\title{
New insights into the mechanism of Schiff base synthesis from aromatic amines in the absence of acid catalyst or polar solvents
}

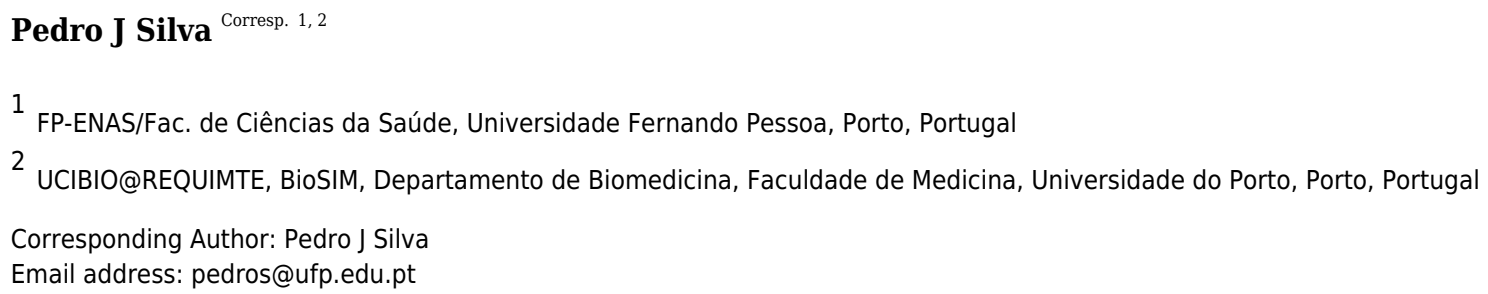

Extensive computational studies of the imine synthesis from amines and aldehydes in water have shown that the large-scale structure of water is needed to afford appropriate charge delocalisation and enable sufficient transition state stabilisation. These insights cannot, however, be applied to the understanding of the reaction pathway in apolar solvents due their inability to form extensive hidrogen-bonding networks. In this work, we perform the first computational studies of this reaction in nonpolar conditions. This density-functional study of the reaction of benzaldehyde with four closely related aromatic amines (aniline, $o$-toluidine, $m$-toluidine and $p$-toluidine) shows that, although an additional molecule of amine may provide some stabilization of the first transition state even in the absence of a hydrogen bonding network, this is insufficient to achieve high reaction rates. Our computations also show that when an extra proton is added to the spectator amine, the activation energies become so low that even picomolar amounts of protonated base are enough to achieve realistic rates. Additional computations show that those minute amounts of protonated base may be obtained under reaction conditions without the addition of extraneous acid through the auto-protolysis of the amines themselves. To our knowledge, this is the first report of a role for the auto-protolysis of anilines in their extensive reactional repertoire. 


\section{Introduction}

3 Imines can be readily synthesized through the reversible reaction of amines with aldehydes. This

4 reaction proceeds through an addition step which forms a carbinolamine intermediate, which is

5 then dehydrated to the imine in the rate-determining step. The released water is usually removed

6 from the system to shift the equilibrium towards the products. The reaction rate is quite sensitive

7 to $\mathrm{pH}$ : moderate amounts of acid greatly accelerate it (Santerre, Hansrote \& Crowell, 1958), but

8 excess acid prevents it (Jencks, 1959 and references therein). The decrease in reaction rate at very

9 low $\mathrm{pH}$ is due to the protonation of the amine, which renders it unable to directly attack the carbonyl, whereas protonation of the carbinolamine is required to achieve high rates of dehydration (Jencks, 1964) (Figure 1). Acid catalysis has also been postulated to proceed through protonation of the carbonyl group, rendering it more susceptible to nucleophilic attack by the amine (Hammett, 1940). The reaction may also take place in the absence of acid catalysis (Law, 1912; Campbell et al., 1948; Crowell \& Peck, 1953), especially with primary amines.

Computational studies of this reaction have shown that in the absence of charge stabilization by solvent the activation energies of the formation of the carbinolamine (Hall \& Smith, 1998; Ding, Cui \& Li, 2015; Ćmikiewicz, Gordon \& Berski, 2018) are prohibitively high (above $25 \mathrm{kcal} \cdot \mathrm{mol}^{-}$ ${ }^{1}$ ) and the activation energies of its dehydration to imine (Hall \& Smith, 1998; Ćmikiewicz, Gordon \& Berski, 2018) are even higher (between 45 and $55 \mathrm{kcal} \cdot \mathrm{mol}^{-1}$ ). Incorporation of one (Hall \& Smith, 1998; Ding, Cui \& Li, 2015) or two (Hall \& Smith, 1998) water molecules as proton transfer assistants greatly facilitates the formation of the carbinolamine by decreasing the activation energy to $8-16 \mathrm{kcal} \cdot \mathrm{mol}^{-1}$ but still affords large barriers incompatible with roomtemperature reaction $\left(26.7 \mathrm{kcal} \cdot \mathrm{mol}^{-1}\right)$ for the dehydration step (Hall \& Smith, 1998). Realistic barriers are, however, obtained when a large number of explicit water molecules (from 9 to 29) are included in the model (Solís-Calero et al., 2012), enabling extensive stabilization of the nascent charges present in the transition state of the dehydration step. Since so far all the computational work on this reaction has been performed on systems including only water as solvent, the aforementioned insights cannot be directly applied to reactions in nonpolar or aprotic solvents, such as the condensation of benzaldehyde with aniline (or toluidines), which is experimentally observed to proceed readily and exothermically in the absence of an acid catalyst (Law, 1912; Campbell et al., 1948; Crowell \& Peck, 1953) or protic solvents. In the 
pathway that affords realistic reaction barrier in the absence of hydrogen-bonding stabilization by protic solvent molecules, and consequently an explanation of how this classic reaction can proceed in nonpolar solvents.

\section{Computational methods}

The geometries of putative intermediates and transition states in the reaction mechanism were optimized using the widely used PBE0 functional (Ernzerhof \& Scuseria, 1999; Adamo \& Barone, 1999), which we have earlier shown to be a very good choice for the description of mechanisms involving the protonation or deprotonation of ketones and amines (Silva \& Ramos, 2011). All geometry optimizations were performed with the Firefly (Granovsky, 2013) quantum chemistry package, which is partially based on the GAMESS (US) (Schmidt et al., 1993) code, using autogenerated delocalized coordinates (Baker, Kessi \& Delley, 1996). In geometry optimizations, the aug-pcseg-1 basis set (Jensen, 2014) was used for heavy atoms and the pcseg-1 basis set was used for hydrogen. Zero-point and thermal effects on the free energies at $298.15 \mathrm{~K}$ were computed at the optimized geometries. DFT energies of the optimized geometries were then computed using the pcseg-2 basis set (Jensen, 2014), which is expected to be close to the complete basis set limit for DFT. The double-hybrid functional DSD-BLYP (Kozuch, Gruzman \& Martin, 2010) supplemented with DFT-D3-BJ corrections (Grimme, Ehrlich \& Goerigk, 2011)was chosen for these single-point energies due to its superlative performance in the computation of total energies vs. the highest quality benchmarks available (Goerigk \& Grimme, 2011; Goerigk et al., 2017). Auto-protolysis constants (pKs) of various amines, ethylene carbonate, acetonitrile, and nitromethane were computed by comparing the energies of separately optimized neutral clusters of each molecule to clusters of the same size which included one single instance of protonated (or deprotonated) molecule. In all cases, intra- and inter-molecular dispersion effects were included in the geometry optimization, frequency calculation, and highlevel single point steps using the DFT-D3 formalism with Becke-Johnson damping developed by Grimme et al. (Grimme et al., 2010; Grimme, Ehrlich \& Goerigk, 2011). Solvation effects in aniline were computed using the Polarizable Continuum Model (Tomasi \& Persico, 1994; Mennucci \& Tomasi, 1997; Cossi et al., 1998) implemented in Firefly. Dispersion and repulsion interactions with the continuum solvent were computed using the method developed by Amovili and Mennucci (Amovilli \& Mennucci, 1997). To obtain estimates of the stability of the postulated bimolecular complexes, the potential of mean force was obtained through umbrella 
64 sampling simulations performed by constraining the distance between the $\mathrm{C}=\mathrm{O}$ in benzaldehyde

65 (or carbinolamine) and the $\mathrm{NH}_{3}{ }^{+}$of anilinium (or $\mathrm{N}-\mathrm{H}$ in a second carbinolamine) with a

66 harmonic potential of the form $V=1 / 2 k\left(x-x_{0}\right)^{2}$ with $k$ equal to $10.0 \mathrm{kcal} / \mathrm{mol} / \AA^{2}$. Molecular

67 dynamics wimulations were performed in YASARA (Krieger \& Vriend, 2015) using the

68 AMBER14 forcefield (Maier et al., 2015). Charge assignment was performed with the AM1BCC

69 protocol (Jakalian et al., 2000; Jakalian, Jack \& Bayly, 2002). Sampling was performed in bins

$70 \quad 0.5 \AA$ apart, for $6 \mathrm{~ns}$ per bin. In each bin, the first full ns was discarded from the analysis. The

71 unbiased distributions were obtained through the weighted histogram analysis method (WHAM)

72 (Kumar et al., 1992; Grossfield, 2018) using a bin size of $0.2 \AA$.

\section{Results}

74 The reactions of benzaldehyde with aniline and its three mono-methylated derivatives ( $o$ -

75 toluidine, $m$-toluidine, and $p$-toluidine) were studied in the gas phase. In all cases, the most stable

76 initial arrangement of aldehyde and the aromatic amine finds both molecules parallel to each

77 other due to the interaction between their aromatic clouds (Figure 2). In the traditional

78 description of this reaction mechanism, the subsequent formation of the carbinolamine

79 intermediate proceeds through the simultaneous attack of the carbonyl carbon atom by the amine

80 lone pair and proton transfer from the amine to the carbonyl oxygen atom. The geometry of this

81 transition state (Figure 2) is virtually identical for the four aromatic amines studied, with a N-C

82 distance of 1.608-1.613 $\AA$, a NH-O distance of 1.384-1.389 $\AA$ and a C-O distance of $1.336 \AA$

83 almost exactly between that of a C-O double bond (1.215 $\AA$ ) and a C-O single bond (1.411 $\AA$ ).

84 The transition states are, however, very hard to reach as they lie $55.4-70.6 \mathrm{kcal} \cdot \mathrm{mol}^{-1}$ above the

85 pre-reactional complex state (Table 1). Since these high activation energies are incompatible with

86 the experimentally observed syntheses of imines from aldehyde and aromatic amines at

87 temperatures between 0 and $60^{\circ} \mathrm{C}$ (Allen \& VanAllan, 1941; Campbell et al., 1948; Crowell \&

88 Peck, 1953), the actual reaction mechanism must be more complex than commonly postulated. 
90 Additional computations showed that the inclusion of an additional molecule of amine greatly

91 facilitates the formation of the carbinolamine by assisting the proton transfer from the amine to

92 the carbonyl oxygen (Figure 3A). The increased energetic stabilization (between 21 and 32

$93 \mathrm{kcal} \cdot \mathrm{mol}^{-1}$, relative to the unaided reaction) yields barriers of $34.4-38.8 \mathrm{kcal} \cdot \mathrm{mol}^{-1}$ above the pre-

94 reactional complex state (Table 2), still far above the values required for detectable reaction rates.

95 The reaction now becomes exergonic (vs. pre-reactional complex) in almost all cases, with the

96 reaction with $o$-toluidine as the sole exception due to the geometric constraints entailed by the

97 close proximity of the methyl substituents in the aromatic amines. Carbinolamine dehydration

98 proved to be difficult (Table 2), with barriers ranging between 28 and $45 \mathrm{kcal} \cdot \mathrm{mol}^{-1}$ relative to the

99 carbinolamine. Previous computational studies of this reaction (Hall \& Smith, 1998; Solís-Calero

100 et al., 2012) showed that this step is also difficult in water models unless large solvent cages are

101 used, which allow very efficient charge delocalization throughout the hydrogen-bonded network

102 (Solís-Calero et al., 2012). Since such stabilization is exceedingly unlikely to be available in

103 aromatic amine solvents due to their inability to form such extended networks, we analysed other

104 possibilities of achieving acceptable reaction rates for the dehydration step. Inspired by the

105 observation of dimeric derivatives of imines obtained through electrochemical reduction (Law,

106 1912) we evaluated the feasibility of stabilizing the carbinolamine dehydration step with a second

107 molecule of carbinolamine. Interaction of two carbinolamines with each other to form a

108 bimolecular pre-reactional complex is energetically very favorable (by $80-95 \mathrm{kcal} \cdot \mathrm{mol}^{-1}$, using

109 PBE0-D3 (BJ)//DSD-BLYP (BJ) in implicit solvent). Umbrella sampling simulations

110 (Supporting Information Figure 1) in a system containing two carbinolamines surrounded by

111325 molecules of aniline confirmed that the formation of a pre-reaction complex composed of

112 two mutually interacting carbinolamines with the correct relative orientations was indeed

113 favorable in the presence of explicit solvent. The subsequent barriers range from 5.2 (for $m$ - 
114 toluidine) to $18-21 \mathrm{kcal} \cdot \mathrm{mol}^{-1}$ (for the other aromatic amines) (Table 3 and Figure 4), well into

115 the range of experimental feasibility, provided that a mechanism for the initial formation of the

116 carbinolamine (that circumvents the high barriers encountered previously) can be found. The

117 feasibility of acid-assisted catalysis was therefore explored. Our computations showed that the

118 addition of one protonated molecule of amine greatly facilitated the atack of the carbonyl by the

119 neutral amine, through the strong stabilization of the nascent negative charge on the carbonyl

120 oxygen (Figure 5). The N-protonated carbinolamine formed in this step may then transfer the

121 extra proton to the assisting base, which in turn funnels it to the leaving hydroxyl group, yielding

122 the protonated Schiff base and a water molecule. For the aliphatic amines tested, the results

123 obtained were not very promising: although the initial formation of the N-protonated

124 carbinolamine did indeed proceed without an energetic barrier, the subsequent proton transfer to

125 the leaving hydroxyl group proved to be quite endergonic due to the relative instability of the

126 produced N-protonated imine. Still, barriers of at most $23 \mathrm{kcal} \cdot \mathrm{mol}^{-1}$ (implying reaction rates of

127 at least $0.3 \mathrm{~h}^{-1}$ ) were obtained in all cases. The height of the barrier decreased markedly when the

128 amine was changed from ethylamine to ethenylamine and ethynylamine, confirming that the

129 introduction of $\pi$-delocalization stabilizes the product (and that the endergonicity of the reactions

130 can be overcome by facilitating the spreading of the anscent positive charge throughout the

131 molecule). In agreement with this interpretation, the reaction barriers obtained (Tables 4 and 5)

132 for the reactions of acetaldehyde or benzaldehyde with the four aromatic amines tested are very

133 small (from 2.5 to $10.5 \cdot \mathrm{kcal} \cdot \mathrm{mol}^{-1}$ ) and therefore have extremely high reaction rates $\left(1.24 \times 10^{5}-\right.$

$1349.1 \times 10^{10} \mathrm{~s}^{-1}$ ). The experimentally observed reaction rates (on the order of $1 \mathrm{~h}^{-1}$ ) can therefore be

135 achieved with minute concentrations of protonated base $\left(10^{-15}-10^{-9} \mathrm{~mol} \cdot \mathrm{dm}^{-3}\right)$.

136 We hypothesized that, even without the addition of acid catalysts, such minute amounts of

137 protonated amine might be available through the auto-protolysis of the amine. Indeed, even some

138 solvents generally regarded as aprotic or only weakly protic have been experimentally shown to 
139 auto-ionize to a limited extent (Mihajlović et al., 1996). To ascertain the likelihood of auto-

140 protolysis of aniline and toluidines, we performed additional computations using small clusters of

141 amine molecules, one of which was kept protonated (or deprotonated). Since very accurate results

142 would require the simulation of very large solvent clusters to account for possible long-range

143 structural rearrangements around the ionized structures, which are unfortunately not possible with

144 our current computational resources, we compared our results with the auto-protolysis constants,

145 computed in the same way, of other solvents which have been studied experimentally. Our results

146 (Table 6) show that the auto-protolysis of most of the amines tested is much more favourable

147 than that of ethylene carbonate $(\mathrm{pKs}=21.5)$ nitromethane $(\mathrm{pKs}=23.7)$ or acetonitrile $(\mathrm{pKs}=28.8)$,

148 and that therefore self-ionization of aniline or toluidines can easily afford concentrations of

149 protonated amine at least as high as $10^{-21.5 / 2}$, which render accessible the mechanism postulated 150 above.

\section{Conclusions}

153 Like the analogous reaction in water (Ding, Cui \& $\mathrm{Li}, 2015$ ), imine formation from 154 benzaldehyde and anilines in nonpolar solvent cannot occur without the intervention of a base 155 which facilitates the transfer of one proton from the amine nitrogen atom to the carbonyl oxygen.

156 Although the energetic stabilization provided by this assistance decreases activation energy by 157 more than $20 \mathrm{kcal} \cdot \mathrm{mol}^{-1}$ relative to the reaction in the gas phase, this is not sufficient to enable 158 reasonable rates of formation of the carbinolamine. The carbinolamine dehydration step is also 159 prohibitively expensive, but can be made more accessible if a bimolecular mechanism (where one 160 carbinolamine catalyzes the dehydration of the other) is taken into account. Both steps can be 161 made much more accessible if the nascent negative charge in the attacked carbonyl (or the 162 leaving hydroxyl in the dehydration step) are stabilized through interaction with the protonated 
163 forms of the reacting bases. Our computations, in turn, show that auto-protolysis of the amines to

164 generate these species is feasible and that the low activation energies of the protonated amine-

165 assisted mechanism fully enable the observation of good reaction rates even from the minute

166 concentrations of protonated amine predicted to exist in water-free aniline/benzaldehyde 167 mixtures.

168

170

171

172

\section{References}

Adamo C., Barone V. 1999. Toward reliable density functional methods without adjustable parameters: The PBE0 model. The Journal of Chemical Physics 110:6158. DOI: 10.1063/1.478522.

Allen CFH., VanAllan J. 1941. m-tolylbenzylamine. Organic Syntheses 21:108. DOI: 10.15227/orgsyn.021.0108.

Amovilli C., Mennucci B. 1997. Self-consistent-field calculation of Pauli repulsion and dispersion contributions to the solvation free energy in the polarizable continuum model. The Journal of Physical Chemistry B 5647:1051-1057.

Baker J., Kessi A., Delley B. 1996. The generation and use of delocalized internal coordinates in geometry optimization. Journal of Chemical Physics 105:192-212. DOI: 10.1063/1.471864.

Campbell KN., Helbing CH., Florkowski MP., Campbell BK. 1948. The Reaction of Grignard Reagents with Schiff Bases. Journal of the American Chemical Society 70:3868-3870. DOI: 10.1021/ja01191a099.

Ćmikiewicz A., Gordon AJ., Berski S. 2018. Characterisation of the reaction mechanism between ammonia and formaldehyde from the topological analysis of ELF and catastrophe theory perspective. Structural Chemistry 29:243-255. DOI: 10.1007/s11224-017-1024-X.

Cossi M., Mennucci B., Pitarch J., Tomasi J. 1998. Correction of cavity-induced errors in polarization charges of continuum solvation models. Journal of Computational Chemistry 19:833-846. DOI: 10.1002/ (sici)1096-987x (199806)19:8<833::aid-jcc3>3.0.co;2-q.

Crowell TI., Peck DW. 1953. Kinetic Evidence for a Schiff Base Intermediate in the Knoevenagel Condensation. Journal of the American Chemical Society 75:1075-1077. DOI: 
193

194

195

196

197

198

199

200

201

202

203

204

205

206

207

208

209

210

211

212

213

214

215

216

217

218

219

220

221

222

223

224

225

Ding YQ., Cui YZ., Li TD. 2015. New views on the reaction of primary amine and aldehyde from DFT study. Journal of Physical Chemistry A 119:4252-4260. DOI: 10.1021/acs.jpca.5b02186.

Ernzerhof M, Scuseria GE. 1999. Assessment of the Perdew-Burke-Ernzerhof exchangecorrelation functional. The Journal of Chemical Physics 110:5029-5036. DOI: 10.1063/1.478401.

Goerigk L., Grimme S. 2011. A thorough benchmark of density functional methods for general main group thermochemistry, kinetics, and noncovalent interactions. Physical Chemistry Chemical Physics 13:6670-6688. DOI: 10.1039/c0cp02984j.

Goerigk L., Hansen A., Bauer C., Ehrlich S., Najibi A., Grimme S. 2017. A look at the density functional theory zoo with the advanced GMTKN55 database for general main group thermochemistry, kinetics and noncovalent interactions. Physical Chemistry Chemical Physics 19:32184-32215. DOI: 10.1039/c7cp04913g.

Granovsky AA. 2013. PC GAMESS/Firefly version 8.0 : http://classic.chem.msu.su/gran/gamess/ index.html.

Grossfield A. 2018. "WHAM: the weighted histogram analysis method."

Grimme S., Antony J., Ehrlich S., Krieg H. 2010. A consistent and accurate ab initio parametrization of density functional dispersion correction (DFT-D) for the 94 elements HPu. The Journal of chemical physics 132:154104. DOI: 10.1063/1.3382344.

Grimme S., Ehrlich S., Goerigk L. 2011. Effect of the damping function in dispersion corrected density functional theory. Journal of Computational Chemistry 32:1456-1465. DOI: $10.1002 /$ jcc. 21759 .

Hall NE., Smith BJ. 1998. High-Level ab Initio Molecular Orbital Calculations of Imine Formation. The Journal of Physical Chemistry A 102:4930-4938. DOI: 10.1021/jp9810825.

Hammett LP. 1940. Physical Organic Chemistry. New York, N. Y.,: McGraw- Hill Book Co., Inc.

Jakalian A, Bush BL, Jack DB, Bayly CI, I ACAM. 2000. Fast, efficient generation of highquality atomic charges. AM1-BCC model: I. Method. Journal of Computational Chemistry 21:132-146. DOI: 10.1002/ (SICI)1096-987X (20000130)21:2<132::AID-JCC5>3.3.CO;2G.

Jakalian A, Jack DB, Bayly CI. 2002. Fast, efficient generation of high-quality atomic charges. AM1-BCC model: II. Parameterization and validation. Journal of Computational Chemistry 23:1623-1641. DOI: 10.1002/jcc.10128. 
226

227

228

229

230

231

232

233

234

235

236

237

238

239

240

241

242

243

244

245

246

247

248

249

250

251

252

253

254

255

256

257

258

Jencks WP. 1959. Studies on the Mechanism of Oxime and Semicarbazone Formation. Journal of the American Chemical Society 81:475-481. DOI: 10.1021/ja01511a053.

Jencks WP. 1964. Mechanism and Catalysis of Simple Carbonyl Group Reactions. Hoboken, NJ, USA: John Wiley \& Sons, Inc. DOI: 10.1002/9780470171813.

Jensen F. 2014. Unifying General and Segmented Contracted Basis Sets. Segmented Polarization Consistent Basis Sets. Journal of Chemical Theory and Computation 10:1074-1085. DOI: 10.1021/ct401026a.

Krieger E, Vriend G. 2015. New ways to boost molecular dynamics simulations. Journal of Computational Chemistry:n/a-n/a. DOI: 10.1002/jcc.23899.

Kozuch S., Gruzman D., Martin JML. 2010. DSD-BLYP: A general purpose double hybrid density functional including spin component scaling and dispersion correction. Journal of Physical Chemistry C 114:20801-20808. DOI: 10.1021/jp1070852.

Kumar S, Bouzida D, Swendsen RH, Kollman PA, Rosenberg JM. 1992. The Weighted Histogram Analysis Method for Free Energy Calculations on Biomolecules: I. The Method. J. Comp. Chem. 13:1011-1021. DOI: http://dx.doi.org/10.1002/jcc.540130812.

Law HD. 1912. XVII.-Electrolytic reduction. Part V. Benzylidene bases. J. Chem. Soc., Trans. 101:154-166. DOI: 10.1039/CT9120100154.

Maier JA, Martinez C, Kasavajhala K, Wickstrom L, Hauser KE, Simmerling C. 2015. ff14SB: Improving the Accuracy of Protein Side Chain and Backbone Parameters from ff99SB. Journal of Chemical Theory and Computation 11:3696-3713. DOI: 10.1021/acs.jctc.5b00255.

Mennucci B., Tomasi J. 1997. Continuum solvation models: A new approach to the problem of solute's charge distribution and cavity boundaries. Journal of Chemical Physics 106:51515158. DOI: $10.1063 / 1.473558$.

Mihajlović R., Simić Z., Mihajlović L., Vukićević M. 1996. Determination of autoprotolysis constants of some non-aqueous solvents using coulometric titration. Talanta 43:2131-2136. DOI: 10.1016/S0039-9140 (96)02000-0.

Santerre GM., Hansrote CJ., Crowell TI. 1958. The Reaction of Aromatic Aldehydes with nButylamine. Acid Catalysis and Substituent Effects. Journal of the American Chemical Society 80:1254-1257. DOI: 10.1021/ja01538a056.

Schmidt MW., Baldridge KK., Boatz JA., Elbert ST., Gordon MS., Jensen JH., Koseki S., Matsunaga N., Nguyen KA., Su S., Windus TL., Dupuis M., Montgomery JA. 1993. General atomic and molecular electronic structure system. Journal of Computational 
Chemistry 14:1347-1363. DOI: 10.1002/jcc.540141112.

260 Silva PJ., Ramos MJ. 2011. Successes and failures of DFT functionals in acid/base and redox reactions of organic and biochemical interest. Computational and Theoretical Chemistry 966:120-126. DOI: 10.1016/j.comptc.2011.02.022. study of the Schiff base formation from acetaldehyde and butylamine, glycine and phosphatidylethanolamine. Theoretical Chemistry Accounts 131:1-12. DOI: 


\section{Table $\mathbf{1}$ (on next page)}

Relative free energies vs. pre-reactional complex $\left(\mathrm{kcal} \cdot \mathrm{mol}^{-1}\right)$ of the species involved in the formation of carbinolamines from benzaldehyde and aniline derivatives

Energies computed at the DSD-BLYP-D3(BJ)/ pcseg2//PBE0-D3(BJ)/(aug)-pcseg1 theory level. Solvation effects in aniline were included with the PCM formalism. 
1 Table 1: Relative free energies vs. pre-reactional complex $\left(\mathrm{kcal} \cdot \mathrm{mol}^{-1}\right)$ of the species

2 involved in the formation of carbinolamines from benzaldehyde and aniline derivatives,

3 computed at the DSD-BLYP-D3(BJ)/pcseg2//PBE0-D3(BJ)/(aug)-pcseg1 theory level.

4 Solvation effects in aniline were included with the PCM formalism.

\begin{tabular}{|c|c|c|c|c|}
\hline & aniline & $o$-toluidine & $m$-toluidine & $p$-toluidine \\
\hline transition state & 55.4 & 63.7 & 70.6 & 62.0 \\
\hline carbaminolamine & 17.8 & 29.1 & 33.8 & 27.5 \\
\hline
\end{tabular}

5 


\section{Table 2 (on next page)}

Relative free energies vs. pre-reactional complex ( $\mathrm{kcal} \cdot \mathrm{mol}-1)$ of the species involved in the base-assisted formation of carbinolamines from benzaldehyde and aniline derivatives

Energies computed at the DSD-BLYP-D3(BJ)/pcseg2//PBE0-D3(BJ)/(aug)-pcseg1 theory level. Solvation effects in aniline were included with the PCM formalism. 
1 Table 2: Relative free energies vs. pre-reactional complex $\left(\mathrm{kcal} \cdot \mathrm{mol}^{-1}\right)$ of the species

2 involved in the base-assisted formation of carbinolamines from benzaldehyde and aniline

3 derivatives, computed at the DSD-BLYP-D3(BJ)/pcseg2//PBE0-D3(BJ)/(aug)-pcseg1 theory

4 level. Solvation effects in aniline were included with the PCM formalism.

\begin{tabular}{|c|c|c|c|c|}
\hline & aniline & $o$-toluidine & $m$-toluidine & $p$-toluidine \\
\hline transition state & 34.4 & 37.8 & 38.8 & 37.1 \\
\hline carbaminolamine & -3.8 & 4.5 & -5.6 & -9.8 \\
\hline $\begin{array}{c}\text { dehydration } \\
\text { transition state }\end{array}$ & 33.7 & 32.9 & 39.8 & 32.0 \\
\hline
\end{tabular}

5 


\section{Table 3 (on next page)}

Relative free energies $(\mathrm{kcal} \cdot \mathrm{mol}-1)$ of the species involved in the bimolecular dehydration of carbinolamines

Energies computed at the DSD-BLYP-D3(BJ)/pcseg2//PBE0-D3(BJ)/(aug)-pcseg1 theory level. Solvation effects in aniline were included with the PCM formalism. 
1 Table 3: Relative free energies $\left(\mathrm{kcal} \cdot \mathrm{mol}^{-1}\right)$ of the species involved in the bimolecular

2 dehydration of carbinolamines, computed at the DSD-BLYP-D3(BJ)/pcseg2//PBE0-

$3 \mathrm{D3}(\mathrm{BJ}) /($ aug)-pcseg1 theory level. Solvation effects in aniline were included with the PCM

4 formalism.

\begin{tabular}{|c|c|c|c|c|}
\hline & aniline & $o$-toluidine & $m$-toluidine & $p$-toluidine \\
\hline $\begin{array}{c}\text { Two interacting } \\
\text { carbinolamines }\end{array}$ & 0.0 & 0.0 & 0.0 & 0.0 \\
\hline $\begin{array}{c}\text { bimolecular transition } \\
\text { state }\end{array}$ & 21.0 & 17.7 & 5.2 & 18.3 \\
\hline $\begin{array}{c}\text { Carbinolamine }+\mathrm{H}_{2} \mathrm{O}+ \\
\text { product }\end{array}$ & 5.2 & -6.9 & -12.2 & -6.2 \\
\hline
\end{tabular}

5

6 


\section{Table 4 (on next page)}

Relative free energies vs. pre-reactional complex $\left(\mathrm{kcal} \cdot \mathrm{mol}^{-1}\right)$ of the species involved in the acid-catalyzed synthesis of imines from acetaldehyde and different amines

Energies computed at the DSD-BLYP-D3(BJ)/pcseg2//PBE0-D3(BJ)/(aug)-pcseg1 theory level. Solvation effects in aniline were included with the PCM formalism. 
1 Table 4: Relative free energies vs. pre-reactional complex $\left(\mathrm{kcal} \cdot \mathrm{mol}^{-1}\right)$ of the species

2 involved in the acid-catalyzed synthesis of imines from acetaldehyde and different amines,

3 computed at the DSD-BLYP-D3(BJ)/pcseg2//PBE0-D3(BJ)/(aug)-pcseg1 theory level.

4 Solvation effects in aniline were included with the PCM formalism.

$\begin{array}{lccccccc} & \begin{array}{c}\text { Pre-reactional } \\ \text { complex }\end{array} & \text { TS1 } & \text { Int0 } & \text { Int1 } & \text { TS2 } & \text { Product } & \begin{array}{c}\text { Highest } \\ \text { barrier }\end{array} \\ \text { methylamine } & 0.0 & 0.7 & -15.4 & -27.7 & -15.1 & -7.5 & \mathbf{2 0 . 2} \\ \text { ethylamine } & 0.0 & -3.4 & -27.7 & -33.3 & -20.9 & -10.3 & \mathbf{2 3 . 1} \\ \text { ethenylamine } & 0.0 & 0.2 & -24.9 & -22.8 & -9.3 & -8.5 & \mathbf{1 6 . 4} \\ \text { ethynylamine } & & 0.0 & -10.3 & -12.7 & -11.8 & -7.7 & \mathbf{4 . 9} \\ \text { aniline } & 0.0 & -1.0 & -7.4 & -9.8 & -5.4 & -11.4 & \mathbf{4 . 5} \\ \text { m-toluidine } & 0.0 & -1.8 & -27.8 & -18.8 & -17.3 & -24.4 & \mathbf{1 0 . 5} \\ \text { o-toluidine } & 0.0 & -4.2 & -21.5 & -23.7 & -15.4 & -19.4 & \mathbf{8 . 3} \\ \text { p-toluidine } & 0.0 & -6.4 & -7.7 & -7.0 & -5.1 & -12.3 & \mathbf{2 . 5}\end{array}$




\section{Table 5 (on next page)}

Relative free energies ( $\mathrm{kcal} \cdot \mathrm{mol}-1$ ) of the species involved in the aromatic aminiumassisted dehydration of the carbinolamines produced from the reaction of benzadelhyde with aromatic amines

Energies computed at the DSD-BLYP-D3(BJ)/pcseg2//PBE0-D3(BJ)/(aug)-pcseg1 theory level. Solvation effects in aniline were included with the PCM formalism. 
1 Table 5: Relative free energies $\left(\mathrm{kcal} \cdot \mathrm{mol}^{-1}\right)$ of the species involved in the aromatic

2 aminium-assisted dehydration of the carbinolamines produced from the reaction of

3 benzadelhyde with aromatic amines, computed at the DSD-BLYP-D3(BJ)/pcseg2//PBE0-

$4 \mathrm{D3}(\mathrm{BJ}) /($ aug)-pcseg1 theory level. Solvation effects in aniline were included with the PCM

5 formalism.

\begin{tabular}{|c|c|c|c|c|}
\hline & aniline & $o$-toluidine & $m$-toluidine & $p$-toluidine \\
\hline $\begin{array}{c}\text { Protonated } \\
\text { carbinolamine + base }\end{array}$ & 0.0 & 0.0 & 0.0 & 0.0 \\
\hline $\begin{array}{c}\text { Carbinolamine + } \\
\text { protonated base }\end{array}$ & 3.2 & 14.0 & 5.0 & 0.8 \\
\hline $\begin{array}{c}\text { dehydration transition } \\
\text { state }\end{array}$ & 7.1 & 19.4 & 14.6 & 8.6 \\
\hline
\end{tabular}

6

7 


\section{Table 6(on next page)}

Computed auto-protolysis energies $\left(\mathrm{kcal} \cdot \mathrm{mol}^{-1}\right)$ of different solvents.

Geometries optimized at the PBE0-D3(BJ)/(aug)-pcseg1 level. Energies computed with DSDBLYP-D3(BJ)/pcseg-2. Solvation effects were included with the PCM formalism. Experimental values were taken from (Mihajlović et al., 1996) 
1 Table 6: Computed auto-protolysis energies $\left(\mathrm{kcal}^{\cdot} \mathrm{mol}^{-1}\right)$ of different solvents. Geometries

2 optimized at the PBE0-D3(BJ)/(aug)-pcseg1 level. Energies computed with DSD-BLYP-

3 D3(BJ)/pcseg-2. Solvation effects were included with the PCM formalism. Experimental

4 values were taken from (Mihajlović et al., 1996)

\begin{tabular}{|c|c|c|}
\hline & Auto-protolysis energies $\left(\mathrm{kcal} \cdot \mathrm{mol}^{-1}\right)$ & $\begin{array}{l}\text { Experimental auto- } \\
\text { protolysis constant }\end{array}$ \\
\hline ethenylamine & 69.4 & n.a. \\
\hline ethynylamine & 72.5 & n.a. \\
\hline$p$-toluidine & 80.7 & n.a. \\
\hline aniline & 82.2 & n.a. \\
\hline$o$-toluidine & 83.4 & n.a. \\
\hline ethylamine & 85.1 & n.a. \\
\hline ethylene carbonate & 88.2 & $10^{-21.5}$ \\
\hline acetonitrile & 95.8 & $10^{-28.8}$ \\
\hline methylamine & 100.9 & n.a. \\
\hline nitromethane & 105.9 & $10^{-23.7}$ \\
\hline$m$-toluidine & 106.1 & n.a. \\
\hline
\end{tabular}


Figure 1

Possible pathways for the reaction of aldehydes with amines

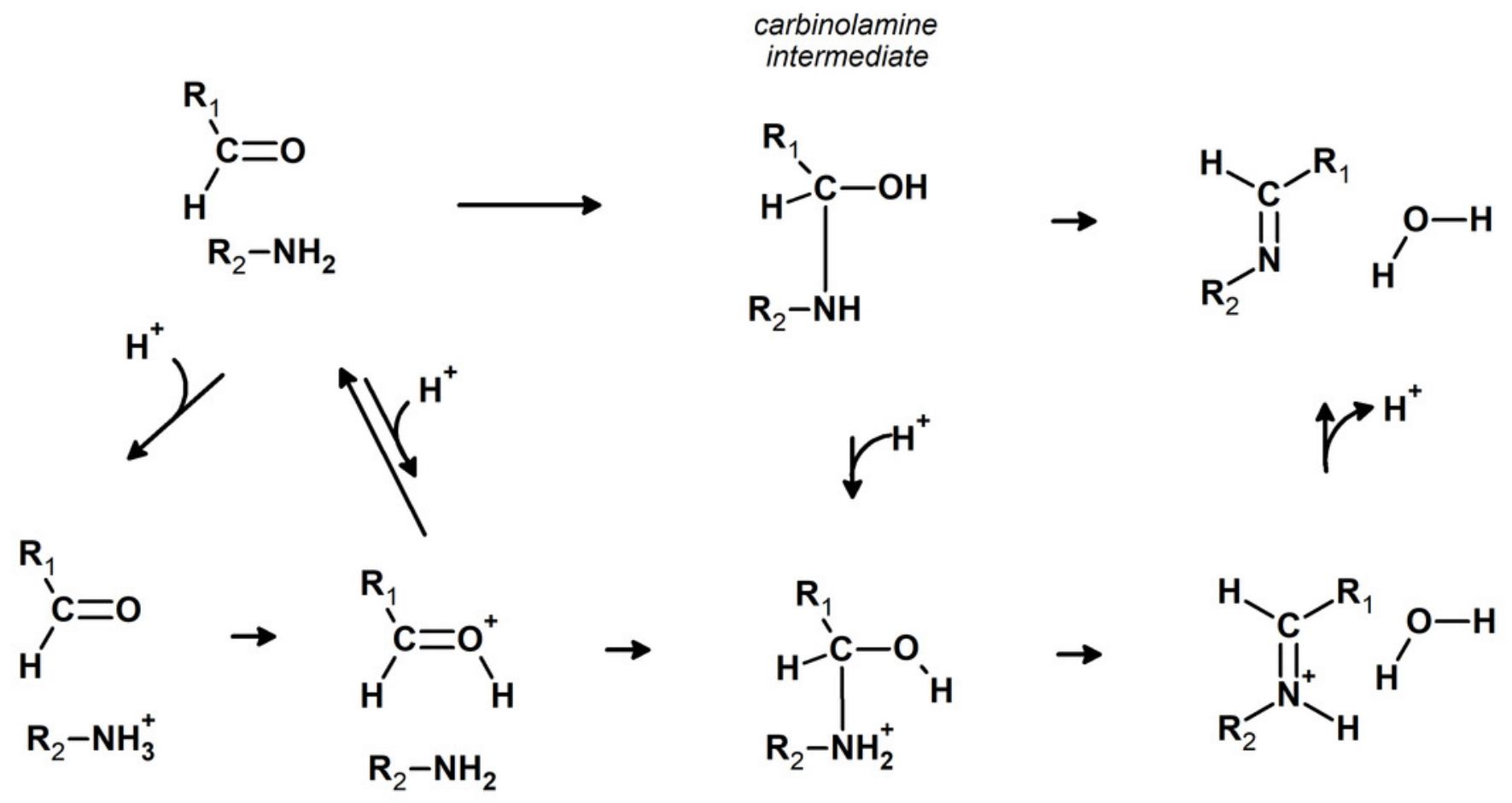


Figure 2

Unassisted formation of carbinolamine.

A) Reactants; B) Transition state; C) Carbinolamine; D) Reaction profile for the unassisted reaction of benzaldehyde with aniline (blue), $m$-toluidine (red), o-toluidine (green) and $p$ toluidine (violet)

A

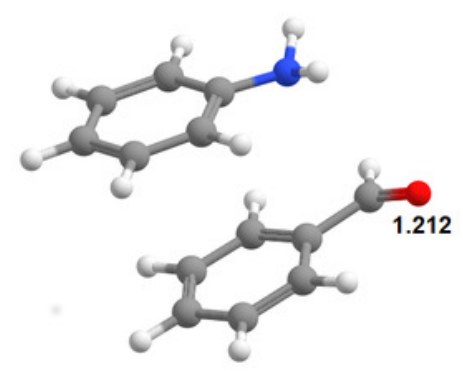

B

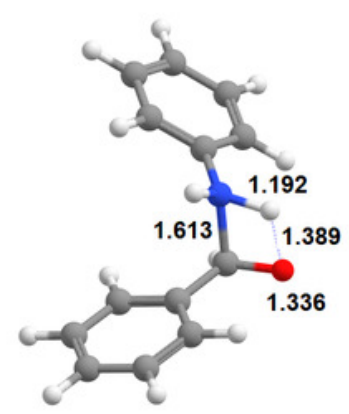

C

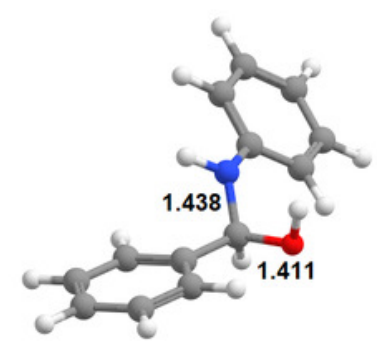

D

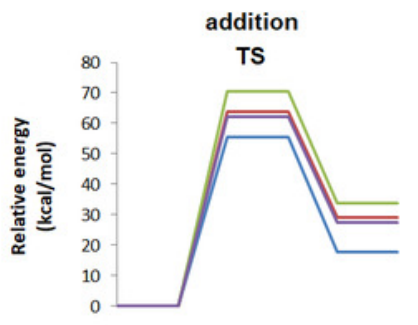


Figure 3

Base-assisted formation of carbinolamine.

A) first transition state; B) carbinolamine and base; C) Transition state of the base-assisted dehydration; D) Reaction profile for the base-assisted reaction of benzaldehyde with aniline (blue), $m$-toluidine (red), o-toluidine (green) and $p$-toluidine (violet)

A

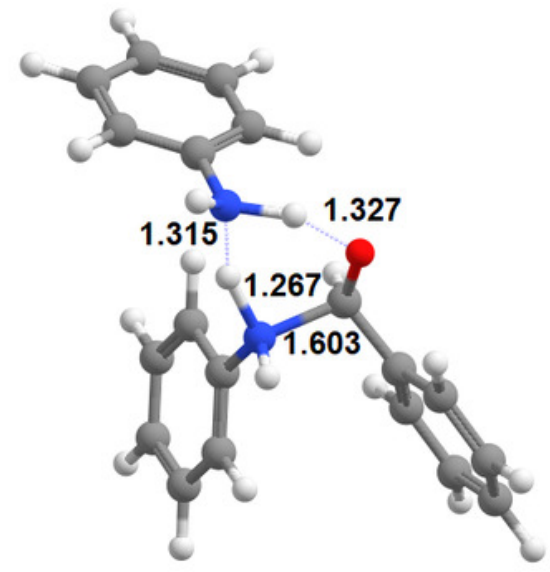

C

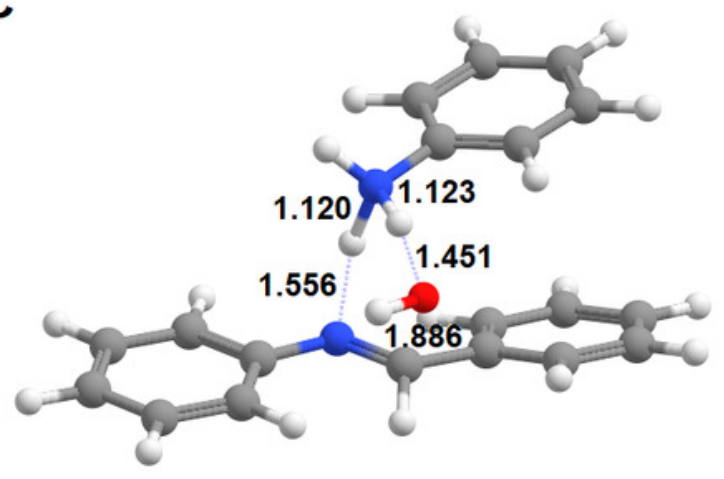

B

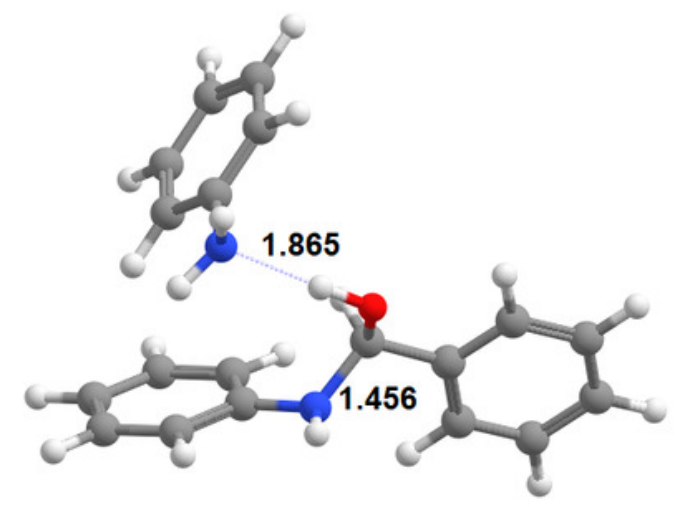

D

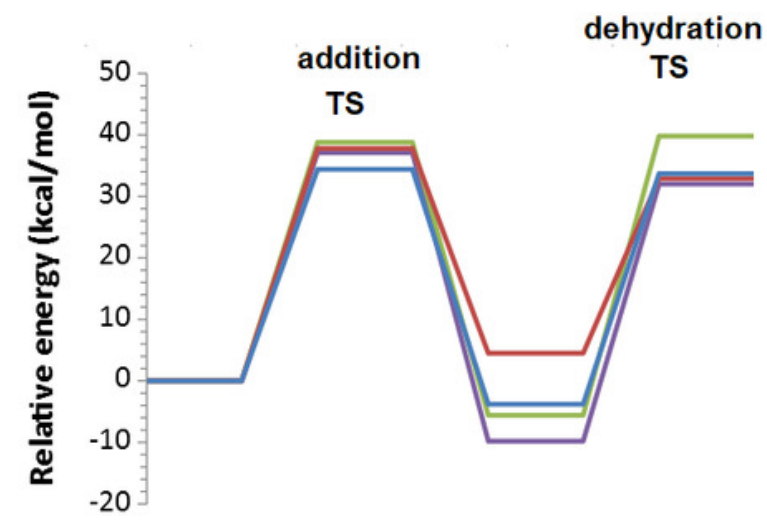


Figure 4

Bimolecular dehydration of carbinolamines.

A) Reaction scheme; B) transition state; C) Potential energy surfaces of the bimolecular dehydration of the carbinolamines produced from the reaction of benzaldehyde with aniline (blue), $m$-toluidine (red), o-toluidine (green) and $p$-toluidine (violet)

A)<smiles>[R2]NC([R])(O)C([R])N[R2]N=C([R])[CH]CC</smiles>

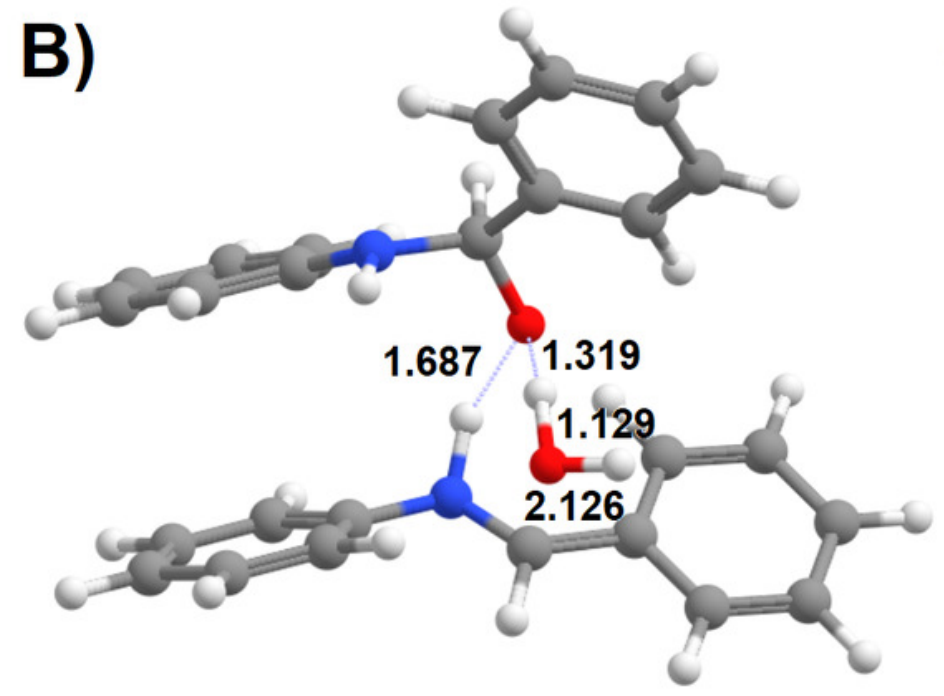

C)

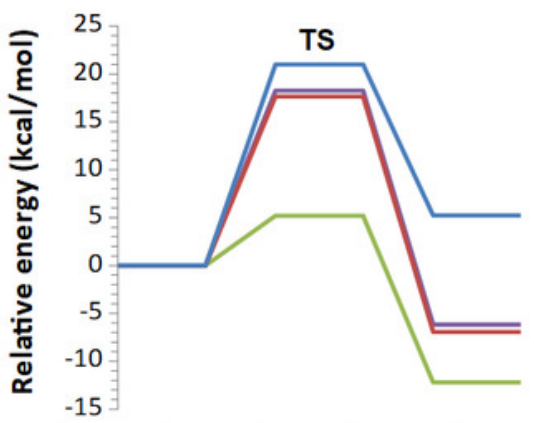




\section{Figure 5}

Reaction profile of the protonated-amine-assisted formation of Schiff bases.

A) N-protonated carbinolamine and neutral amine; B) carbinolamine and protonated amine;

C) transition state of the dehydration step; D) Potential energy surfaces of the reactions of acetaldehyde with methylamine (blue), ethylamine (red), ethenylamine (green) and ethynylamine (violet); E) Potential energy surfaces of the reactions of acetaldehyde with aniline (blue), $m$-toluidine (red), o-toluidine (green) and $p$-toluidine (violet)

A)

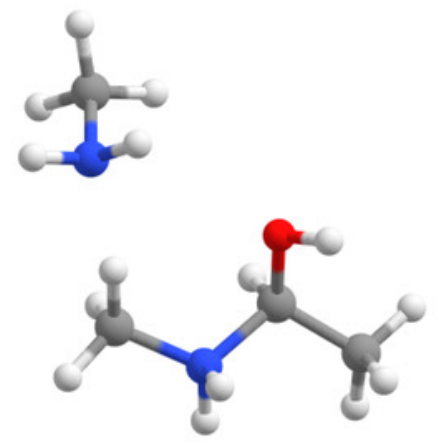

B)
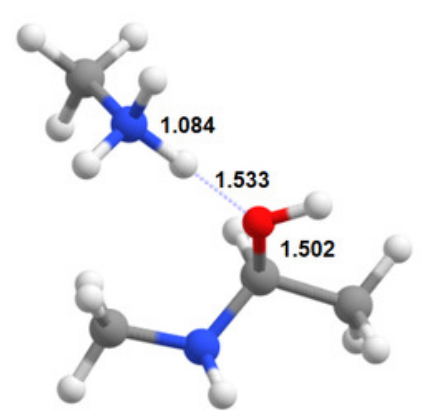

C)

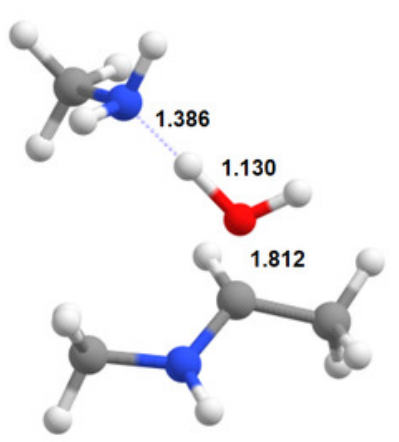

D)

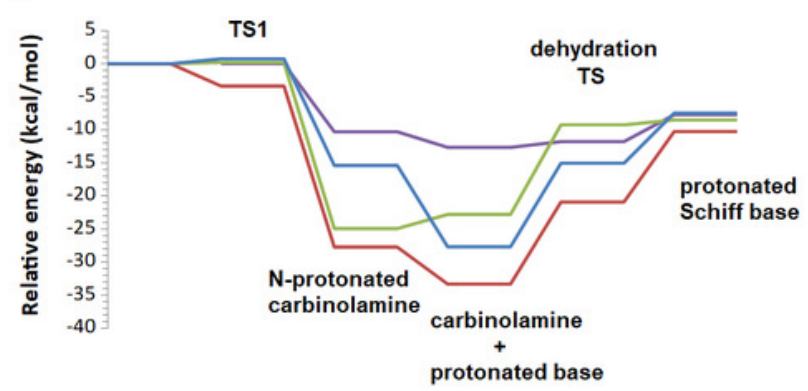

E)

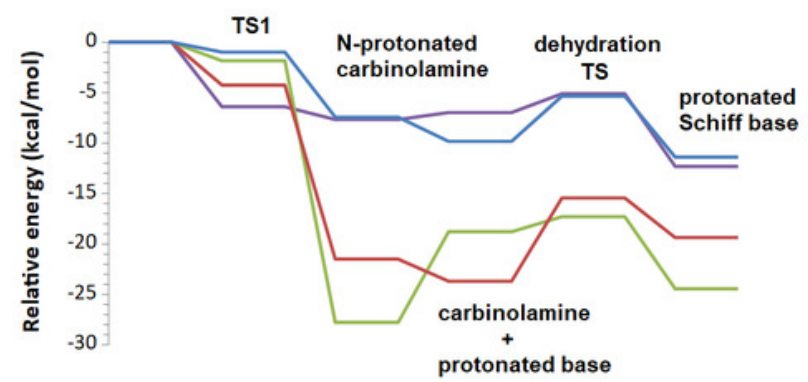

